\title{
Investigation of Variable-Diffusion Turbulence Model Correction for Round Jets
}

\author{
William A. Engblom ${ }^{1}$ \\ The Aerospace Corporation, Cleveland, Ohio \\ Nicholas J. Georgiadis ${ }^{2}$ \\ NASA, Glenn Research Center, Cleveland, Ohio \\ and \\ Abbas Khavaran ${ }^{3}$ \\ QSS Group Inc., Glenn Research Center, Cleveland Ohio
}

\begin{abstract}
A Reynolds-Averaged Navier-Stokes (RANS) correction is described to account for a key effect of acoustic interaction near the end of the potential core for axisymmetric jets. Specifically, the Variable-Diffusion (Var-D) correction is formulated to mimic the effect of increased shear layer instability/motion near the end of the potential core by adjusting the local diffusion of $k$ and $\varepsilon$. The proposed Var-D correction is initially evaluated for an unheated axisymmetric jet with a perfectly expanded jet Mach number of 0.5 . Results for centerline velocity distribution and turbulent kinetic energy contours using commonly employed turbulence models (i.e. SST and $k-\varepsilon$ ) and $k-\varepsilon$ with the Var-D correction are compared. Substantial improvement for prediction of potential core length, centerline velocity decay, and $k$ field is demonstrated with the Var-D correction. The sensitivity of the proposed model to the closure coefficients associated with the correction is also examined using this cold subsonic jet case. Similar turbulence model performance comparisons are made for three other jets to evaluate the sensitivity of the correction to increased jet exhaust compressibility and heated jet flow conditions. The effect of the improved mean and turbulence field predictions obtained using the Var-D correction on farfield noise prediction is also evaluated using the JeNo acoustic analogy code, and shown to be negligible. Although the approach holds promise, the applicability of the Var-D correction is currently limited to round jets.
\end{abstract}

\section{Nomenclature}

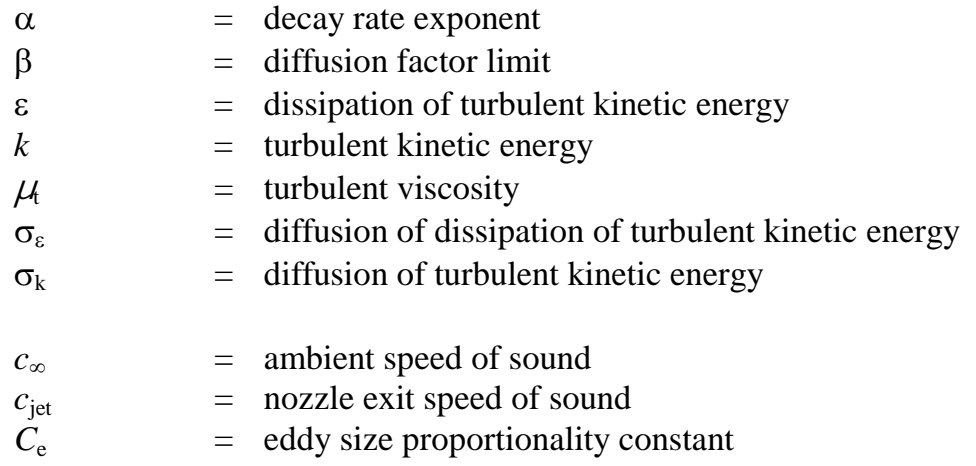

\footnotetext{
${ }^{1}$ Engineering Specialist, Fluid Mechanics, 21000 Brookpark Rd, Mail 86-7, Senior Member AIAA.

${ }^{2}$ Senior Research Scientist, Nozzle Branch, 21000 Brookpark Rd, Mail 86-7, Associate Fellow AIAA.

${ }^{3}$ Research Engineer, Acoustics Branch, 21000 Brookpark Rd, Senior Member AIAA.
} 


$\begin{array}{ll}D & =\text { nozzle exit diameter } \\ L_{e} & =\text { eddy size length scale } \\ M_{\mathrm{a}} & =\text { acoustic Mach number, }=U_{\text {jet }} / c_{\infty} \\ M_{\text {jet }} & =\text { jet exit Mach number, }=U_{\text {jet }} / c_{\text {jet }} \\ r & =\text { radial location } \\ R & =\text { radial location of local idealized eddy } \\ S P D & =\text { Sound spectral density } \\ S t & =\text { Strouhal number } \\ T_{\mathrm{r}} & =\text { exhaust-to-ambient static temperature ratio } \\ u & =\text { axial velocity } \\ U_{\text {jet }} & =\text { jet exit axial velocity } \\ x & =\text { axial distance from nozzle exit plane }\end{array}$

\section{Introduction}

Despite the routine use Reynolds-averaged Navier-Stokes (RANS) techniques for analysis of aerospace systems, the accurate prediction of nozzle and jet flows remains an area of needed improvement. Turbulence modeling remains the pacing item limiting the accuracy of jet flow predictions. Further, for aeroacoustics analysis, both the mean flow and turbulence state are important for assessment of noise emitted by jets under consideration. While Large-Eddy Simulation (LES) offers promise for the future by resolving the large-scale unsteady turbulent motion, this technique is currently prohibitively expensive computationally for realistic, high Reynolds number flows. Hence, RANS approaches will be required for the foreseeable future. As a result, there is still a need for work in the area of RANS turbulence modeling for jets, including turbulence model development to more accurately calculate developing jet flow features, as well as for comprehensive assessment of modeling advances to determine capabilities and limitations.

Effective use of RANS computational fluid dynamics (CFD) in nozzle aeroacoustics investigations requires accurate simulation of both the mean flow and turbulent kinetic energy fields. Capturing the initial jet growth region remains a difficulty. There are many examples of subsonic jet potential core length underprediction in the literature. For example, Koch et. al [1] investigated subsonic axisymmetric separate flow jets with three flow solvers using twoequation $k-\varepsilon$ turbulence models where the mixing rate in each of the calculations was slower than that indicated by experimental results. The turbulent kinetic energy levels were also lower, which corresponds to the slower mixing rate. Engblom et. al [2] investigated a series of cold and hot single flow subsonic nozzle flows including a baseline round nozzle and several chevron nozzles, and a similar trend in the computations indicated much slower mixing towards the nozzle centerline than observed in experiments. Georgiadis et. al [3] investigated a reference subsonic lobed nozzle flow with linear two-equation and explicit algebraic stress turbulence models, and found similar trends. These and many other studies suggests that the propagation of turbulence to the jet centerline is actually more rapid than that predicted by standard two-equation RANS turbulence models. This results in the calculations producing longer jet potential core lengths than experimentally measured. Additionally, far downstream of the end of the jet potential core, it has been generally found that computed farfield mixing rate becomes too high. As a result, currently available RANS turbulence models are not adequate for accurate prediction of jet flow details.

The impetus for the current investigation comes from detailed comparisons of the mean velocity and $k$ fields predicted by the Wind code against Particle Imaging Velocimetry (PIV) data [2] that suggest that the RANS deficiency is most evident in the vicinity of the end of the potential core. More specifically, RANS predicts the shear layer to remain well-defined even as it reaches the centerline at a shallow angle, leaving a relatively thin "sliver" of core flow; whereas experimental results indicate that the shear layer is strongly diffused at the end of the potential core. We suggest that this deficiency is largely related to important missing fluid physics: the effect of acoustic interaction between merging portions of the shear layer.

Steady-state RANS models are inherently unable to account for the effect of acoustic interaction and feedback in many aeroacoustics flows. Further, the basis of RANS modeling is to replace the unsteady nature of turbulent flows with effective turbulent stresses. For the two-equation models used here, the turbulent stress is 
related to the mean strain rate by an effective eddy (or turbulent) viscosity. In RANS, turbulence is primarily generated via the mean shear stresses present in boundary and shear layers in a jet flow. The effect of acoustic feedback on large-scale instability and fine-scale turbulence levels cannot be addressed without a detailed timedependent calculation. For example, a Mach 0.8 jet impinging a nearby wall was found to generate at least $20 \mathrm{~dB}$ higher broadband noise at almost all frequencies than in the absence of the wall [4]. This enhanced mixing is related to the feedback tone, and cannot be addressed in a steady-state RANS computation. It follows that jet flows which involve the merging of a shear layer could produce acoustic interactions and feedback that are not resolvable by RANS.

Unsteady RANS (URANS) simulations have also not proven to be accurate for aeroacoustics problems in which capturing acoustic feedback is vital. For example, Shih and Hamed [5] found that for flow over an open cavity, URANS was unable to capture the unsteadiness, resulting in steady-flow results due to the excessive turbulent viscosities present. They also found that URANS did not adequately capture the coupling of acoustic and turbulence effects. Our attempts to apply URANS to round jets at moderate and high subsonic speeds have also typically produced firmly steady-state results. Although Detached eddy Simulation (DES) and LES approaches have been shown to resolve the unsteadiness in such flows [6], the present work is interested in a production-type computing capability, and thus is restricted to consideration of the RANS approach.

\section{Methodology}

We propose a RANS correction to account for the effect of acoustic interaction near the end of the potential core. The design of the correction is described and shown to have its roots in fluid physics. We evaluate and calibrate the correction against detailed measurements for a subsonic cold stream nozzle flow (set point \#3) from the Acoustic Reference Nozzle (ARN) database [7], including PIV and rake data. The correction is configured to produce the correct centerline velocity decay (and potential core length) for cold subsonic round jets, while improving the accuracy of the velocity profile downstream of the potential core and improving the accuracy of the $k$ field predictions. We also evaluate the model correction against data for other set points (SP) to investigate the sensitivity of correction accuracy to compressibility and heated-jet exhaust conditions. Table 1 consists of the run parameters for each of the ARN cases that are evaluated, including exit Mach number, acoustic Mach number, and static temperature ratio.

\section{Table 1. Run matrix selected from ARN database}

\begin{tabular}{|c|c|c|c|}
\hline $\mathrm{SP}$ & $M_{\text {iet }}$ & $M_{\mathrm{a}}$ & $T_{\mathrm{r}}$ \\
\hline$\# 3$ & 0.51 & 0.5 & 0.95 \\
\hline$\# 7$ & 0.98 & 0.9 & 0.84 \\
\hline$\# 23$ & 0.38 & 0.5 & 1.76 \\
\hline$\# 27$ & 0.68 & 0.9 & 1.76 \\
\hline
\end{tabular}

We utilized the Wind code [8], version 5, for all computations presented herein. The Wind code is provided by the NPARC Alliance, a partnership between NASA Glenn Research Center, The Boeing Company, and Arnold Engineering Development Center (AEDC).

\section{Proposed Variable-Diffusion (Var-D) Correction}

We propose a correction to the standard Chien $k-\varepsilon$ model [9] to roughly account for the effect of acoustic interaction near the end of the potential core. Specifically, we will formulate a correction to the local diffusion of $k$ and $\varepsilon$ to mimic the effect of increased shear layer instability/motion near the end of the potential core. The high Reynolds number form of the $k-\varepsilon$ equations and definition of turbulent viscosity $\left(\mu_{t}\right)$ are shown below (Eq. 1). The closure coefficients specific to the standard Chien model are also listed. 


$$
\begin{aligned}
& \frac{D(\rho k)}{D t}=\frac{\partial}{\partial x_{j}}\left[\left(\mu+\frac{\mu_{t}}{\sigma_{k}}\right) \frac{\partial k}{\partial x_{j}}\right]+\tau_{i j} \frac{\partial u_{i}}{\partial x_{j}}-\rho \varepsilon \\
& \frac{D(\rho \varepsilon)}{D t}=\frac{\partial}{\partial x_{j}}\left[\left(\mu+\frac{\mu_{t}}{\sigma_{\varepsilon}}\right) \frac{\partial \varepsilon}{\partial x_{j}}\right]+C_{\varepsilon 1} \frac{\varepsilon}{k} \tau_{i j} \frac{\partial u_{i}}{\partial x_{j}}-C_{\varepsilon 2} \rho \frac{\varepsilon^{2}}{k} \\
& \text { Where the turbulent viscosity is given by: } \quad \mu_{t}=C_{\mu} \rho \frac{k^{2}}{\varepsilon} \\
& \text { Chien's closure coefficients are: } C_{\mu}=0.09, \sigma_{k}=1.0, \sigma_{\varepsilon}=1.3, C_{\varepsilon 1}=1.35 \text {, and } C_{\varepsilon 2}=1.8 .
\end{aligned}
$$

The merging of the shear layer at the end of the potential core clearly involves complex acoustic and fluid dynamic interactions. One can imagine a feedback loop such that the violent merging of the shear layer results in acoustic radiation which, in turn, effects the shear layer development prior to the merge. One can also imagine that the interaction would include acoustic radiation across the narrow potential core that could lead to increased fluid dynamic unsteadiness and mixing across the potential core. The physics of such interactions cannot be addressed by RANS formulations. Consequently, for the proposed correction, it is hypothesized that acoustic interaction across the potential core is most significant where we have large energetic eddies within the shear layer in close proximity (see Fig. 1). So, we assume the missing interaction effect is driven by a proximity ratio (Eq. 2 ) of the local eddy size $\left(L_{e}\right)$, or radial extent, to the radial distance $(R)$ from the centerline (i.e., half the distance from local eddy to a mirror image eddy). Note that we limit the value of the proximity ratio to unity since it is not physically reasonable for $L_{e}$ to be larger than $R$ and overlap the centerline.

$$
P R(\text { Proximity Ratio })=\min \left\{\left(L_{e} / R\right), 1\right\}
$$

The local eddy size (Eq. 3) is assumed proportional to the local turbulent mixing length, $L_{t}=C_{D} * k^{1.5} / \varepsilon$ [10]. That is,we expect the eddy length proportionality constant $\left(C_{e}\right)$ to be of the same order as the constant in the turbulent mixing length expression $\left(C_{D} \sim 0.164\right)$. We initially set this constant to 0.5 , and later examine the sensitivity to values between 0.5 and 1.0 .

$$
L_{e}=C_{e} * k^{1.5} / \varepsilon
$$

The acoustic influence of each local eddy is assumed to decay with decreasing proximity ratio according to an acoustic factor (Eq. 4). The decay rate $(\alpha)$ for the rms pressure fluctuations radiated from a turbulent shear layer can be shown analytically to be inversely proportional to the distance from the source to the farfield observer [11]. However, in the near-field of a large local eddy it is no longer appropriate to assume such behavior. Consequently, we arbitrarily chose a stronger cubic decay rate $(\alpha=3)$, and will examine the sensitivity to weaker decay rates (i.e., quadratic and linear decays).

$$
A F(\text { Acoustic Factor })=P R^{\alpha}
$$

As mentioned earlier, we propose to mimic the motion of the shear layer near the end of the potential core by adding diffusion to the $k$ and $\varepsilon$ fields. Specifically, we chose to increase the diffusion (i.e., reduce $\sigma_{k}$ and $\sigma_{\varepsilon}$ ) for each equation based on the same diffusion factor (Eq. 5). For example, the $\sigma_{k}$ and $\sigma_{\varepsilon}$ values are permitted to vary between 1 and 4 times smaller than the standard Chien values when $\beta=0.25$. Note that this correction is only having an effect close to the centerline and where there are significant gradients in $k$ and $\varepsilon$ to diffuse (i.e., in vicinity of the end of the potential core). The baseline form of the correction, used for all computations herein, is provided as Eq. 6. A simpler alternative form that enforces the same total variation in diffusion factor and provides similar results is also provided as Eq. 7.

$$
\sigma_{k, \varepsilon}(\text { modified })=\sigma_{k, \varepsilon} * D F
$$


Where DF (Diffusion Factor $)=\max (\beta, 1-\min (1, A F))$

\section{$\underline{\text { Var-D Correction (Baseline Formulation) }}$}

$$
\begin{aligned}
& \sigma_{k, \varepsilon \text { (modified) }}=\sigma_{k, \varepsilon} \max [\beta, 1-\min (1, A F)] \\
& \text { where, } \mathrm{AF}=\left(C_{e} \frac{k^{3 / 2} / \varepsilon}{R}\right)^{\alpha} ; \text { AF limited to } 1, C_{e}=0.5, \alpha=3, \beta=0.25
\end{aligned}
$$

\section{$\underline{\text { Var-D Correction (Alternative Formulation) }}$}

$$
\sigma_{k, e \text { (modified })}=\sigma_{k, e} \frac{(1+\mathrm{AF})}{(1+\mathrm{AF} / \beta)}
$$

where, $\mathrm{AF}=\left(C_{e} \frac{k^{3 / 2} / \varepsilon}{R}\right)^{\alpha} ; C_{e}=0.5, \alpha=3, \beta=0.25$

\section{Results}

\section{$\underline{\text { Evaluation of Var-D Correction for Jet SP\#3 }}$}

We begin the evaluation of the proposed RANS correction, using the baseline values of the coefficients described previously (i.e., $C_{e}=0.5, \alpha=3, \beta=0.25$ ), for an unheated axisymmetric jet from Ref. [7] with a perfectly expanded jet Mach number of 0.5. This relatively slow speed case was selected in order to avoid any significant compressibility effects. Comparison of PIV data from this Mach 0.5 jet case with hot-wire measurements from a Mach 0.3 round jet investigated in reference [12] indicated very similar normalized mean flow and turbulence statistics, which verifies that the current Mach 0.5 case under consideration will not be noticeably biased by compressibility effects. In addition to the baseline Chien k- $\varepsilon$ model and the modified k- $\varepsilon$ model using the Var-D correction, we also examine the Menter Shear-Stress Transport (SST) model (Refs. 13 and 14) in the initial comparisons discussed here. This model uses a k- $\omega$ model in near wall regions and a k- $\varepsilon$ model transformed to a $k-\omega$ set of equations away from walls and in the mixing regions.

A comparison of centerline axial velocities using the three turbulence models is provided in Fig. 2. The SST model indicates a potential core that is substantially longer that the experimental data. The unmodified Chien $\mathrm{k}-\varepsilon$ model also provides a potential core that is too long while the modified k- $\varepsilon$ solution obtained with the Var-D correction enables prediction of a shorter potential core that is in very close agreement with the experimental PIV measurements. Note that further downstream, the SST and unmodified k- $\varepsilon$ model results have a mixing rate that is faster than the experimental data or the modified $k-\varepsilon$ results. Centerline turbulent kinetic energy profiles shown in Fig. 3 correspond to the potential core lengths indicated by Fig. 2. That is, the Var-D correction enables a faster transport of turbulent kinetic energy to the centerline than for the unmodified $k-\varepsilon$ model. This is perhaps more effectively observed in the turbulent kinetic energy contours shown in Fig. 4. The experimental PIV data indicates a strongly diffused shear layer at the end of the potential core. Only the modified k- $\varepsilon$ solution reproduces this rapid diffusion of the shear layer as observed through these turbulent kinetic energy contours. Further details of axial velocity and turbulent kinetic energy profiles (shown in Figs. 5 and 6 respectively) also indicate that the primary deficiency of the unmodified models is near the jet centerline. This is particularly evident in the downstream turbulent kinetic energy profiles in Fig. 6. 


\section{$\underline{\text { Sensitivity to Var-D Correction Coefficients }}$}

The initial results using the modified model are quite encouraging when examining the very close agreement of the model with experimental data for the mean centerline axial velocity decay shown in Fig. 2. Qualitative improvement over the unmodified models for enabling the rapid diffusion of turbulent kinetic energy near the end of the potential core is also found in the contours. However, it is necessary to examine the sensitivity of the proposed model to the closure coefficients associated with the correction and also to try to improve the agreement of the centerline turbulent kinetic energy along the centerline.

First we examine effects of $C_{e}$ used in the proximity ratio defined previously. Fig. 7 shows that varying this proportionality constant between 0.5 and 1.0 does not have a significant effect on the mean axial velocity decay. However, there is a significant effect on the turbulent kinetic energy, as evidenced by the centerline profiles in Fig. 8 and the contours in Fig. 9. In particular, it may be noted that if $C_{e}$ is chosen to be too large this causes the variable diffusion effect to propagate further from the centerline and the peak turbulent kinetic energy contours in the shear layer are reduced too much when compared to the experimental data.

Next we consider the acoustic factor decay exponent, $\alpha$. The default value chosen for the coefficient was 3 , corresponding to a cubic decay rate. Here we also indicate quadratic and linear decay rates, obtained by setting the exponent on the proximity ratio to 2 and 1 respectively. We hold $C_{e}$ fixed at an intermediate value of 0.67 for these decay rate investigations. Examining effects of the acoustic factor exponent on the centerline velocity development in Fig. 10, the position of the end of the potential core is not highly dependent on the exponent. Fig. 11 shows a decrease in the peak centerline turbulent kinetic energy as the exponent is reduced. Finally, Fig. 12 shows that when the decay rate becomes too small, the turbulent kinetic energy away from the centerline is reduced too much in comparison to the experimental data.

\section{Continued Evaluation using ARN Data (SP\#7, SP\#23, SP\#27)}

It was previously shown that the baseline Var-D correction significantly improves the predicted mean flow and turbulence in low-speed unheated jets (i.e. SP\#3). Next we examine the effect of jet speed and temperature in order to determine if similar improvements can be detected at other conditions. The baseline set of coefficients $\left(C_{e}\right.$ $=0.5, \alpha=3, \beta=0.25$ ) are used for each run with Var-D. Figs. 13 and 14 show the influence of Var-D on the centerline values of the mean axial velocity and the turbulent kinetic energy at SP\#7 (near-sonic, unheated jet). The centerline velocity decay (Fig. 13) appears rather unaffected by the correction. The $k$ predictions show better agreement at the peak, however, the initial rise in turbulence level appears to diverge from data and the standard k- $\varepsilon$ predictions. Similar comparisons are also shown at SP\#23 (low-subsonic, heated jet). As with the baseline cold jet, the centerline velocity decay (Fig. 15) shows significant improvement when the Var-D correction is applied. Fig. 16 indicates that there is also a substantial improvement in the centerline distribution of the $k$, both in the location of the peak and its magnitude. Finally, Fig. 17 demonstrates an overall improvement in the $k$ distribution in the vicinity of the ending of the potential core as a result of the Var-D corrections. Figs. 18 and 19 illustrate the centerline axial velocity and the tke distributions at SP\#27 (higher-speed, heated jet). The same favorable improvements are predicted with the Var-D correction. These results suggest that the correction is not effective for near-sonic conditions, but appear to be effective in both cold and heated jet flows at low to moderately subsonic conditions.

\section{Influence of Improved Flowfield Prediction on Jet Noise Prediction}

A main motivation behind this effort was to determine if a more accurate flowfield prediction in the vicinity of a round jet centerline could significantly affect the farfield noise prediction. Since the Var-D correction provided a substantial improvement to the jet flowfield prediction, it makes sense to evaluate the sensitivity of the farfield noise prediction to this improvement. The JeNo code [15], based on the linearized form of Lilley's equation, is utilized to predict jet noise spectral directivities for the two sets of RANS solutions involving cold jets (SP\#3 and SP\#7). JeNo uses the mean flow solutions to drive the propagation Green's function in a locally parallel flow. The RANS solutions also provide the time- and length-scales needed to calculate the source; i.e., the two-point spacetime correlation of the turbulent velocity components. These correlations are modeled assuming an isotropic turbulence and using the exponential temporal and spatial functions.

Figs. 20 and 21 display the narrow-band spectral density (SPD) versus Strouhal number ( $\mathrm{St}=\mathrm{f} \mathrm{D} / U_{\text {jet }}$ ) using JeNo jet noise prediction code combined with the Wind RANS solutions. Predictions are presented in lossless form on an arc at 100D, and are compared with the ARN microphone measurements at SP\#3 and SP\#7 respectively. Data 
is plotted at observer inlet angles of $90^{\circ}$ and $150^{\circ}$. The comparisons indicate that the impact of the correction, versus the uncorrected $\mathrm{k}-\varepsilon$ on the predicted noise spectra is small over the entire frequency range at both angles considered (i.e. less than $1 \mathrm{~dB}$ ). This is reasonable to expect since the farfield noise prediction is driven by the $k$ source integration of the turbulence over the entire jet volume, and is dominated by the source strength at and along the jet lip-line. The relatively small region near the end of the potential core does not represent enough source volume to have an important impact. It should also be pointed out that these JeNo predictions agree remarkably well with the cold jet experimental data.

\section{Conclusions}

The proposed correction has been developed to mimic the experimentally observed rapid mixing and $k$ diffusion that occurs near the end of the potential core in a round jet via variable diffusion of $k$ and $\varepsilon$. Traditional RANS modeling simply cannot address such complex acoustic/fluid dynamics interactions. Applying the variable diffusion (Var-D) correction results in significant improvement to flowfield prediction in the vicinity of the centerline for cold and heated subsonic round jets. The correction improved the diffusion of turbulent kinetic energy to the centerline near the end of the jet potential core and resulted in a closer agreement between predictions and the measurements on the centerline. As expected, the improvement in flowfield did not have an appreciable effect on the predicted farfield noise spectra via the JeNo code.

Although the proposed correction is limited to the round jets, the methodology seems promising and a more general approach is under consideration for aeroacoustics applications. A related application for the proposed correction, which requires accurate representation of the jet plume, might include an exhaust plume signature prediction.

\section{Acknowledgements}

This work was funded by the Quiet Aircraft Technology (QAT) program at NASA Glenn Research Center under contract C-31035-T. The authors are grateful to Dr. James Bridges for providing all experimental data, and Dr. Dennis Yoder for significant technical contributions.

\section{References}

1. Koch, L.D., Bridges, J. E., and Khavaran, A., "Flowfield Comparisons From Three Navier-Stokes Solvers for an Axisymmetric Separate Flow Jet," AIAA Paper 2002-0672.

2. Engblom, W. A., Khavaran, A., and Bridges, J.E., "Numerical Prediction of Chevron Nozzle Noise Using Wind-MGBK Methodology,” AIAA Paper 2004-2979, May 2004.

3. Georgiadis, N.J., Rumsey, C.L., Yoder, D.A., and Zaman, K.B. M. Q., "Effects of RANS Turbulence Modeling on Calculation of Lobed Nozzle Flowfields," AIAA Paper 2003-1271, Jan. 2003.

4. Ahuja, K.K., "The Role of the Feedback Phenomenon in Many Aeroacoustics Problems of Current Interest (Invited Paper), ” AIAA Paper 2001-0077, Jan. 2001.

5. Shih S. H, Hamed, A, Yeuan J. J., "Unsteady Supersonic Cavity Flow Simulations Using Coupled KappaEpsilon and Navier-Stokes Equations," AIAA Journal, Vol. 32, No. 10, 1994, pp. 2015-2021.

6. Hamed, A., Basu, D., Das, K., "Detached Eddy Simulations of Supersonic Flow Over Cavity," AIAA Paper 2003-0549, Jan. 2003.

7. Bridges, J.E. and Wernet, M.P., "Measurements of the Aeroacoustic Sound Source in Hot Jets," AIAA Paper 2003-3130, May 2003.

8. Nelson, C.C. and Power, G.D., "CHSSI Project CFD-7: The NPARC Alliance Flow Simulation System," AIAA Paper 2001-0594, Jan. 2001.

9. Chien, K.-Y., "Predictions of Channel and Boundary Layer Flows with a Low-Reynolds-Number Turbulence Model," AIAA Journal, Vol. 20, No. 1, 1982, pp. 33-38.

10. Anderson, D., Tannehill, J., and Fletcher, R., Computational Fluid Mechanics and Heat Transfer, Hemisphere Publishing Co., 1984, p. 230-1.

11. Goldstein, M.E., Aeroacoustics of Flight Vehicles: Theory and Practice, NASA Reference Pub 1258, Vol. 1, Chapter 5, p. 300-303, 1991. 
12. Zaman, K.B.M.Q., Wang, F.Y., and Georgiadis, N.J., "Noise, Turbulence, and Thrust of Subsonic Free Jets from Lobed Nozzles," AIAA Journal, Vol. 41, No. 3, 2003, pp. 398-407.

13. Menter, F.R., "Two-Equation Eddy-Viscosity Turbulence Models for Engineering Applications," AIAA Journal, Vol. 32, No. 8, 1994, pp. 1598-1605.

14. Menter, F.R., “Zonal Two-Equation k- $\omega$ Turbulence Models for Aerodynamic Flows, AIAA Paper 93-2906, July 1993.

15. Khavaran, A., and Bridges, J, “Modeling of Turbulence Generated Noise in Jets,” AIAA Paper 2004-2983, June 2004.

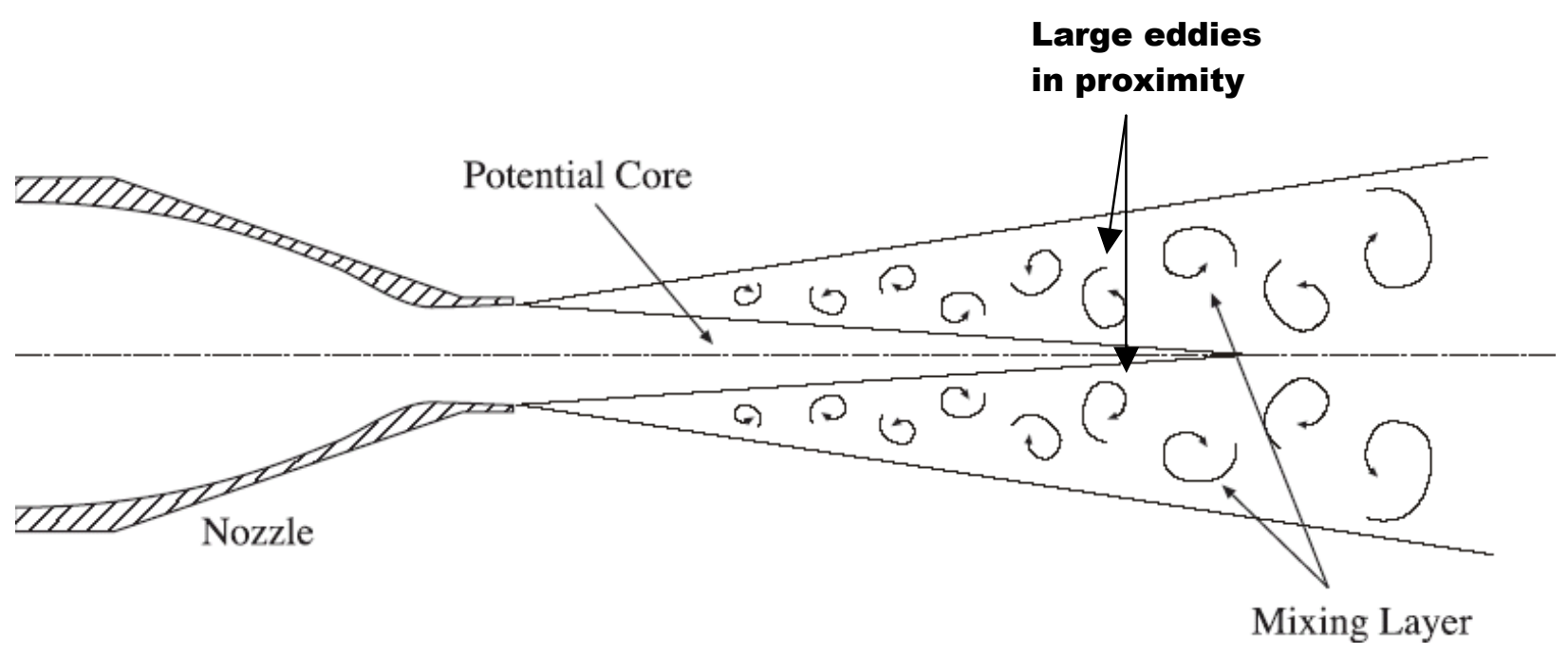

Fig. 1. Schematic of jet flowfield 


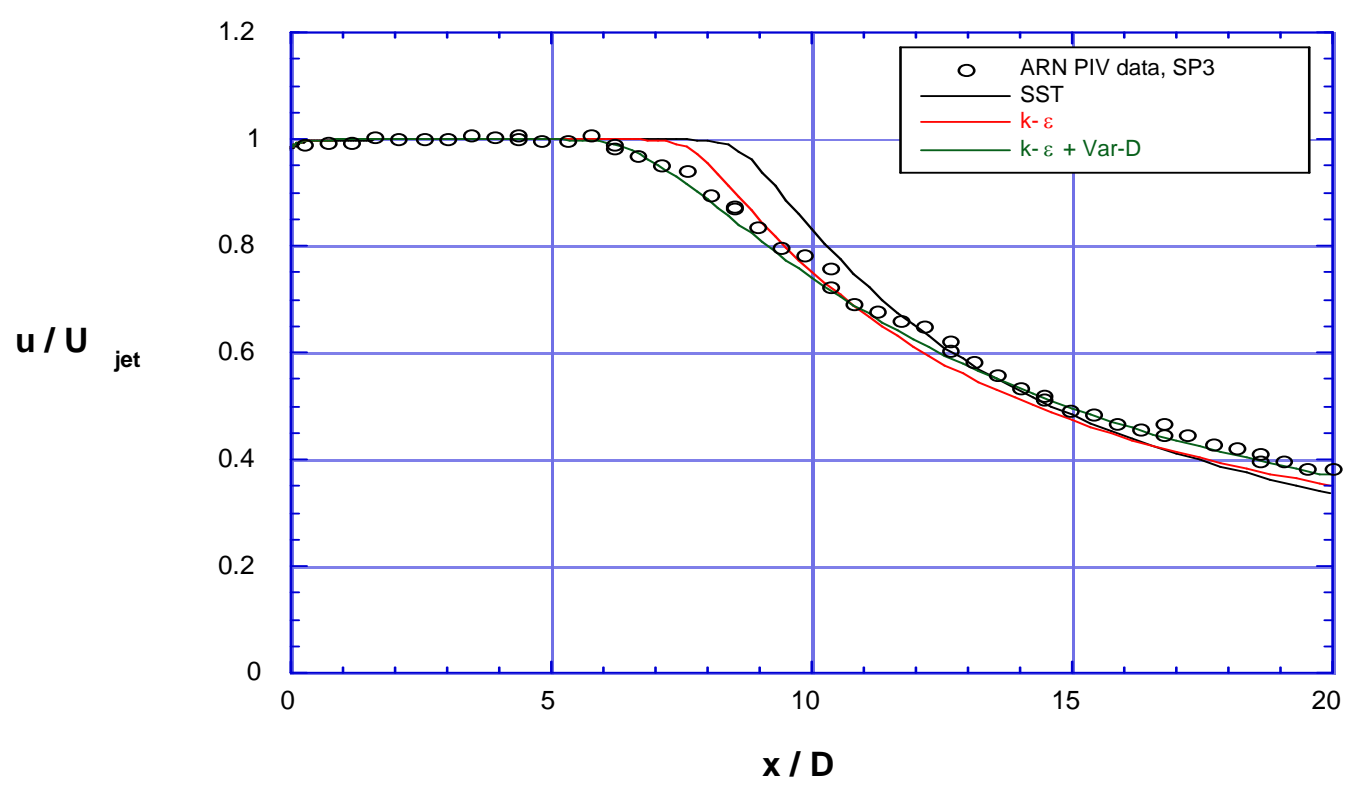

Fig. 2. Centerline axial velocity decay profiles with turbulence model variations.

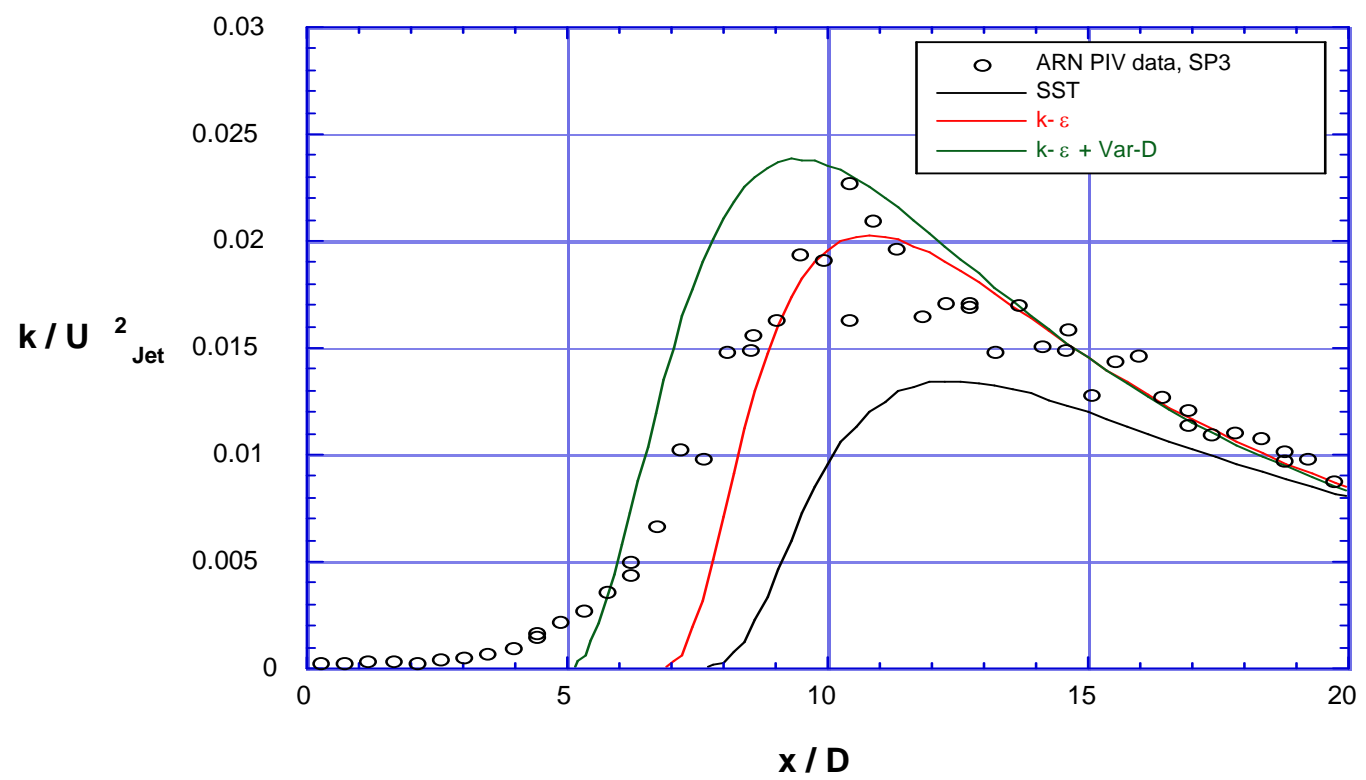

Fig. 3. Centerline turbulent kinetic energy profiles with turbulence model variations. 


\section{$\mathrm{k} / \mathrm{U}^{2}$ jet Contours \\ 0.000 \\ 0.035}

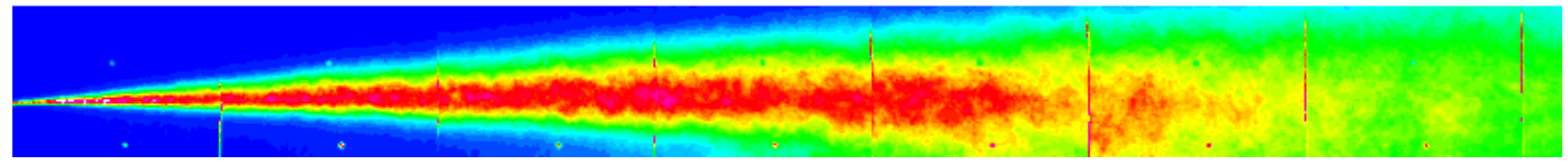

(a) ARN PIV data

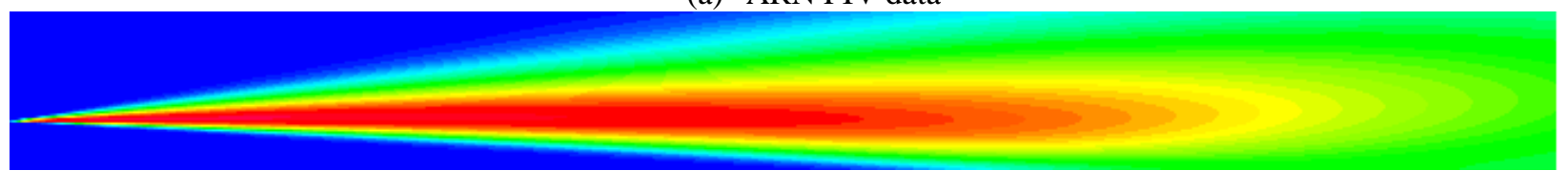

(b) Wind - SST solution

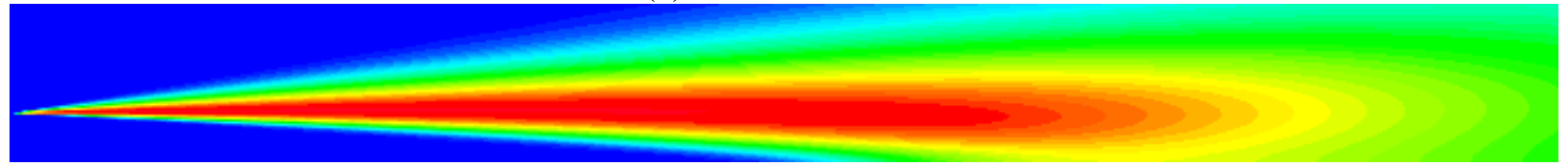

(c) Wind - k- $\varepsilon$ solution

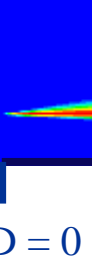

(d) Wind $-\mathrm{k}-\varepsilon+$ Var-D solution

$\mathrm{x} / \mathrm{D}=15$

Fig. 4. Turbulent kinetic energy contours with turbulence model variations.

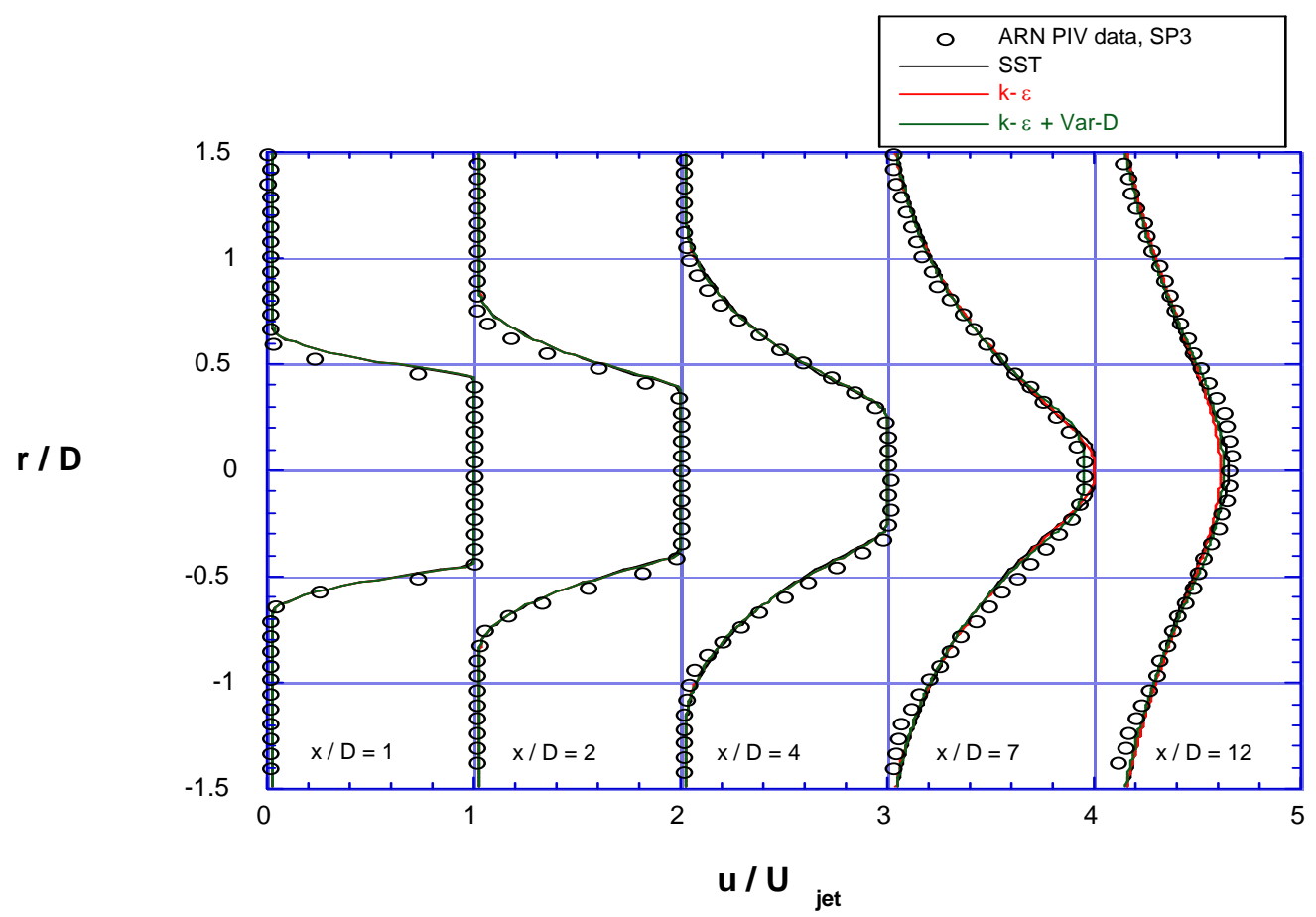

Figure 5. Axial velocity profiles. 


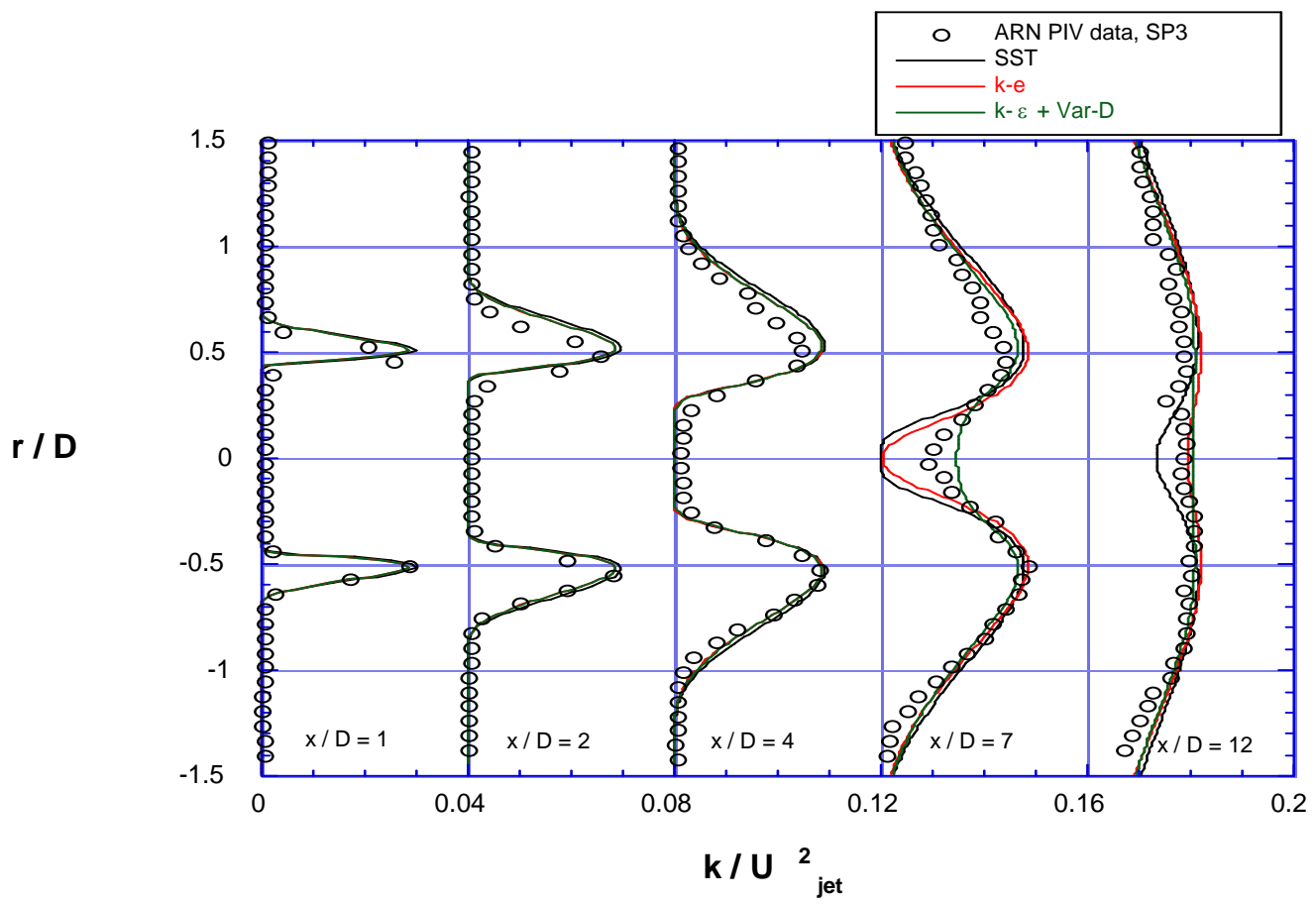

Fig. 6. Turbulent kinetic energy profiles.

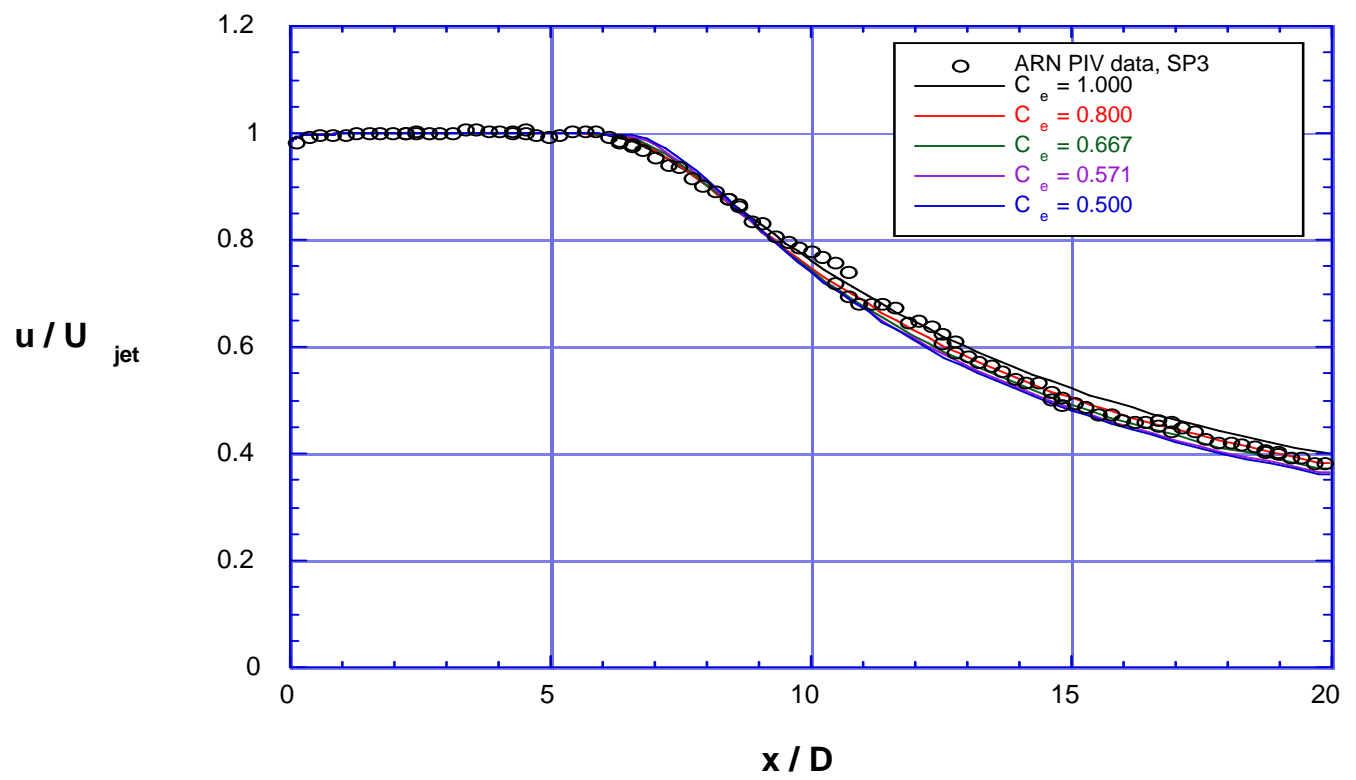

Fig. 7. Centerline axial velocity - variations with eddy size proportionality constant. 


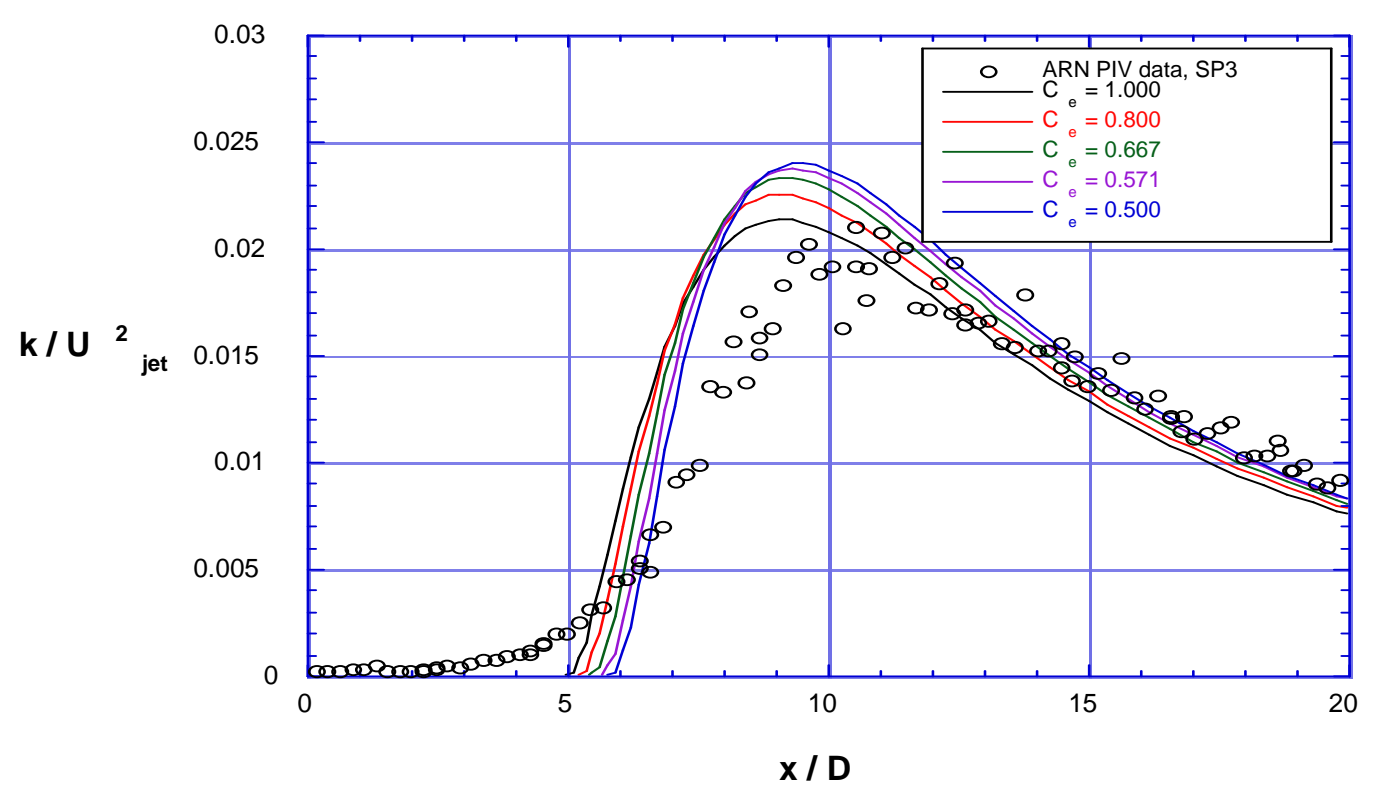

Fig. 8. Centerline turbulent kinetic energy - variations with eddy size proportionality constant.

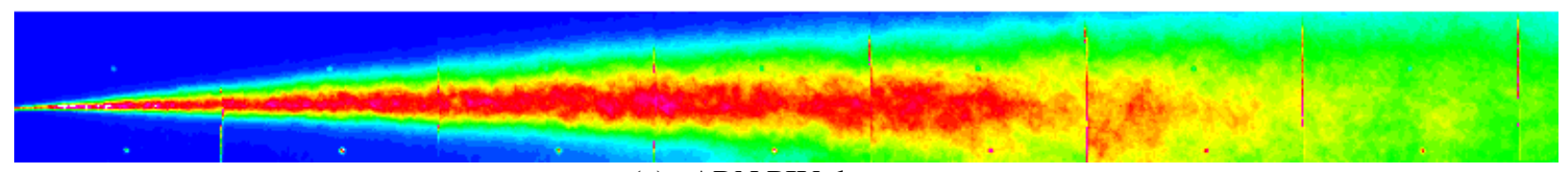

(a) ARN PIV data

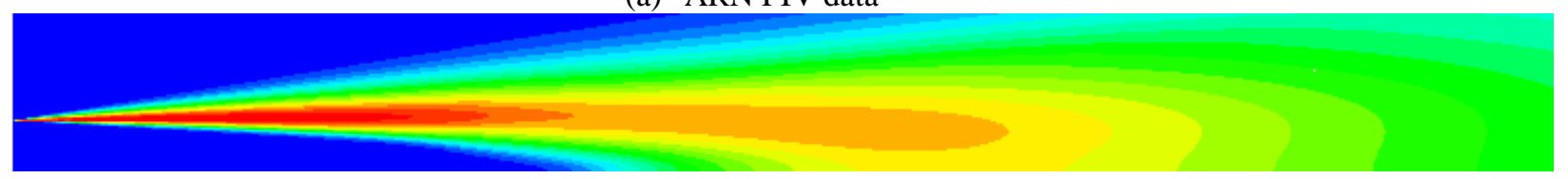

(b) $\mathrm{C}_{\mathrm{e}}=1.0$

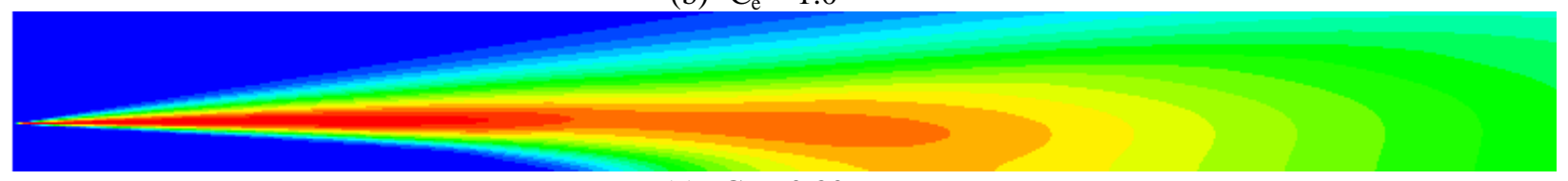

(c) $\mathrm{C}_{\mathrm{e}}=0.80$

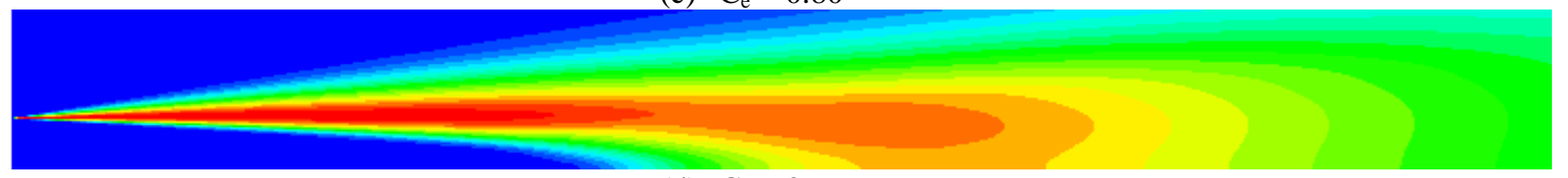

(d) $\mathrm{C}_{\mathrm{e}}=0.67$

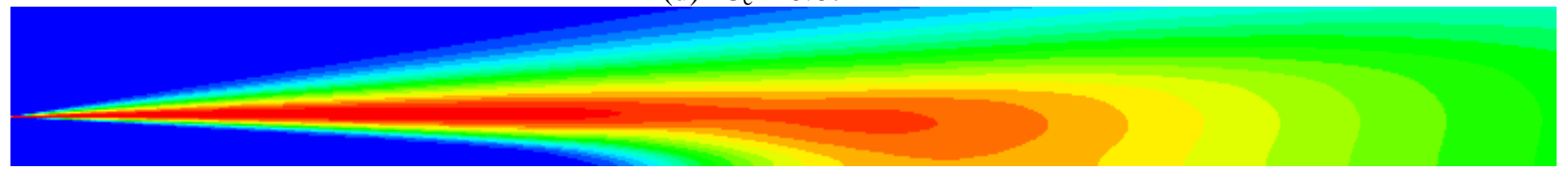

(e) $\mathrm{C}_{\mathrm{e}}=0.57$

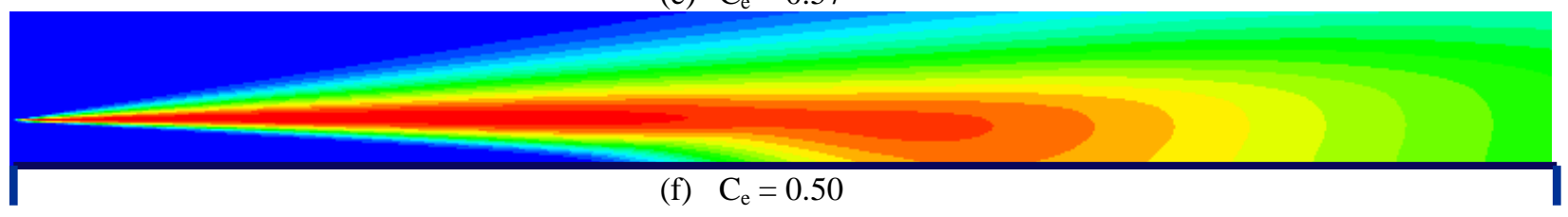

$\mathrm{x} / \mathrm{D}=0 \quad$ Fig. 9 . Turbulent kinetic energy profiles - variation with eddy size proportionality constant 


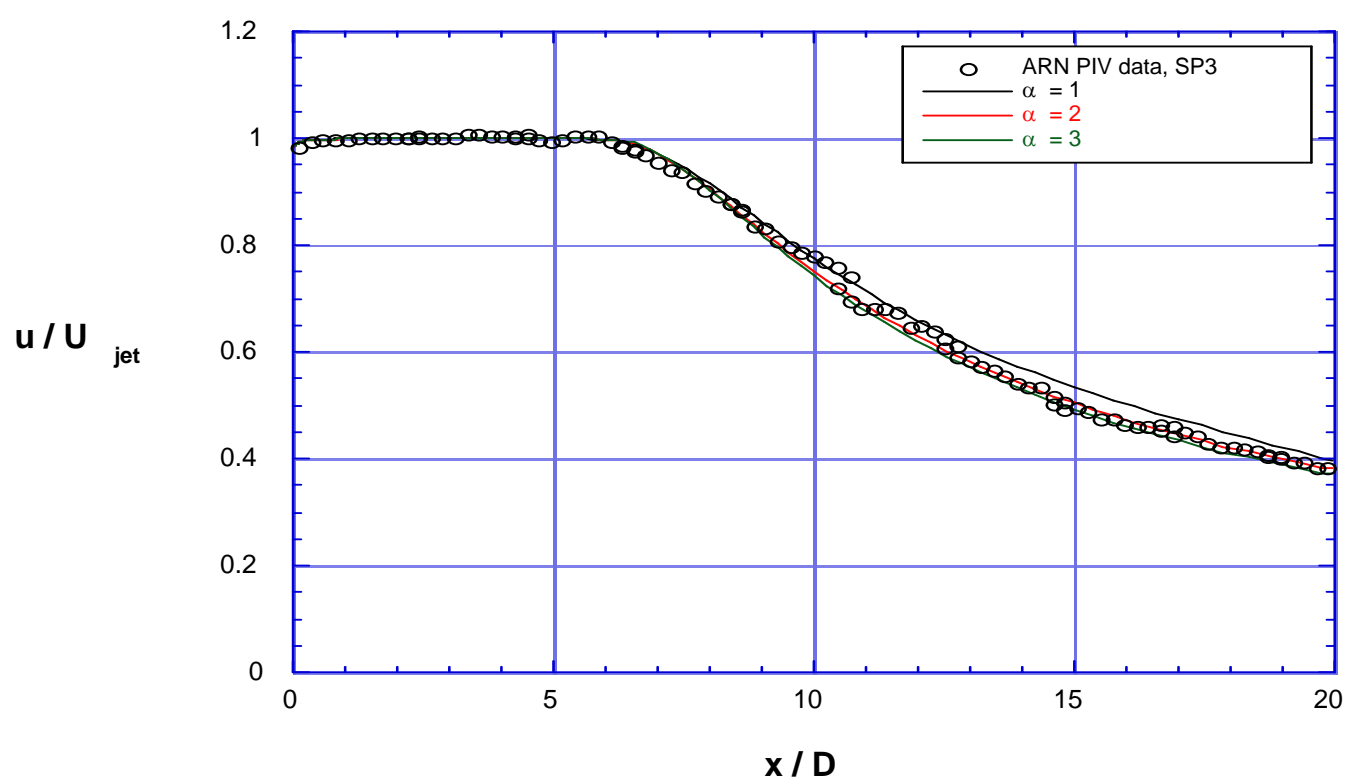

Fig. 10. Centerline axial velocity - variations with decay rate exponent.

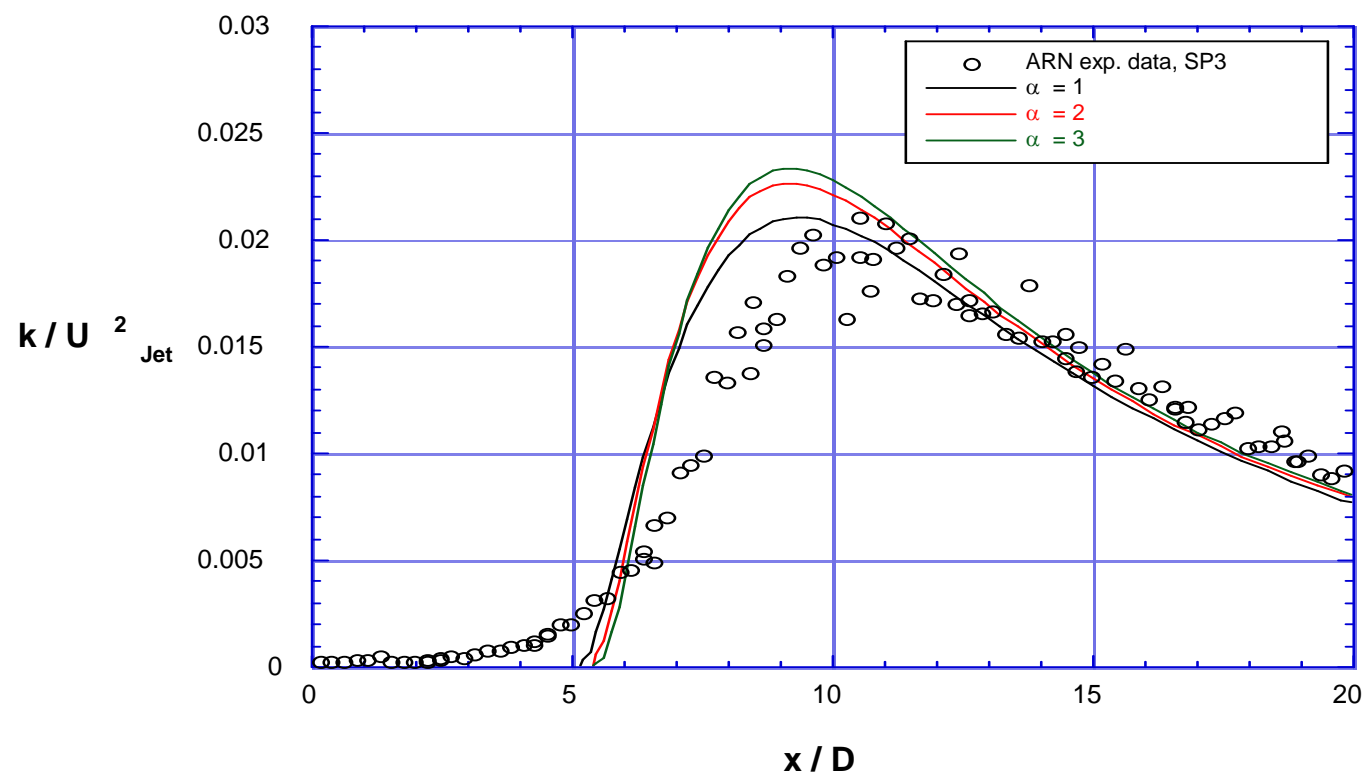

Fig. 11. Centerline turbulent kinetic energy - variations with decay rate exponent. 


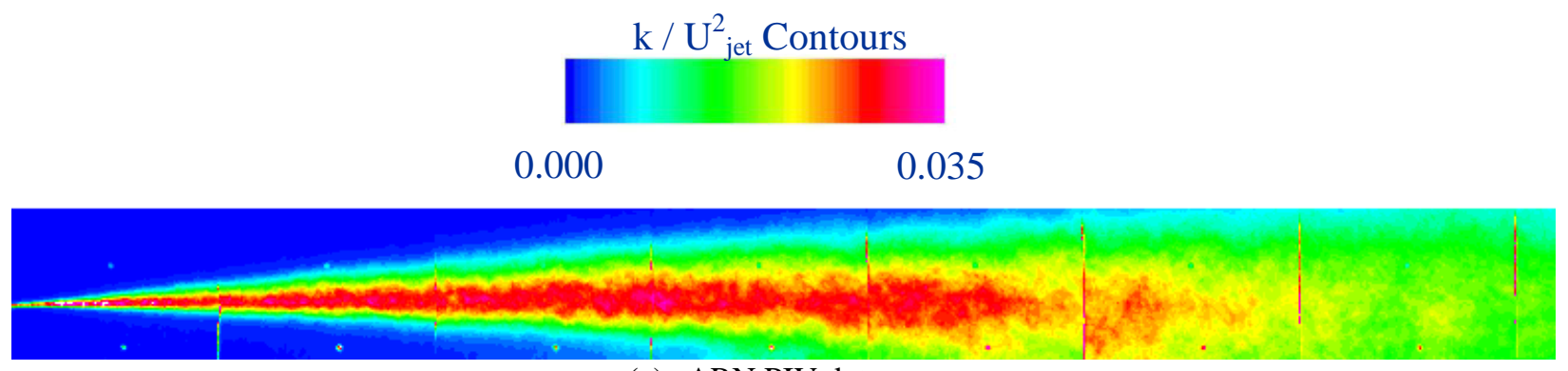

(a) ARN PIV data

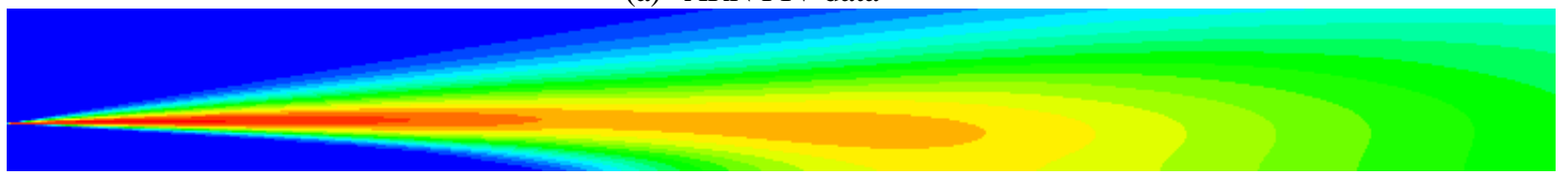

(b) $\alpha=1.0$

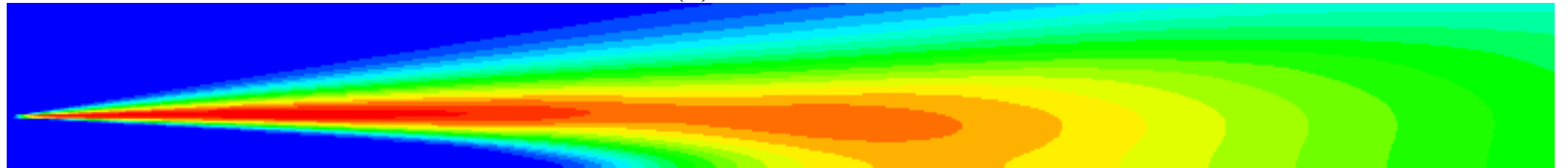

(c) $\alpha=2.0$

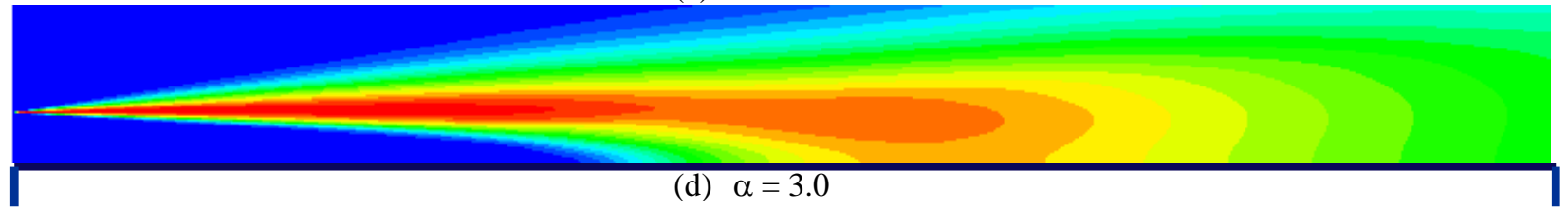

$\mathrm{x} / \mathrm{D}=0$

Fig. 12. Turbulent kinetic energy profiles - variation with decay rate exponent.

$\mathrm{x} / \mathrm{D}=15$ 


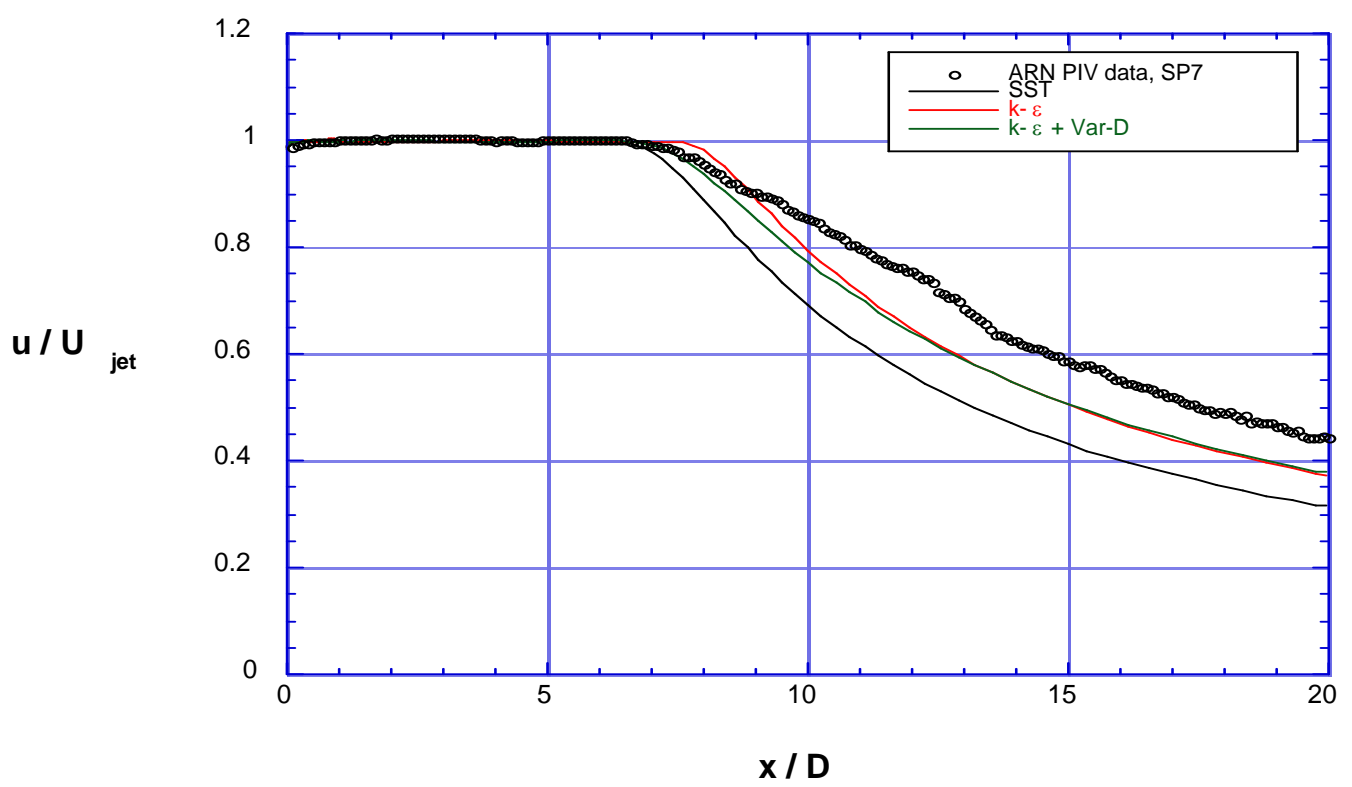

Fig. 13. Centerline axial velocity for SP7 (near-sonic, unheated jet).

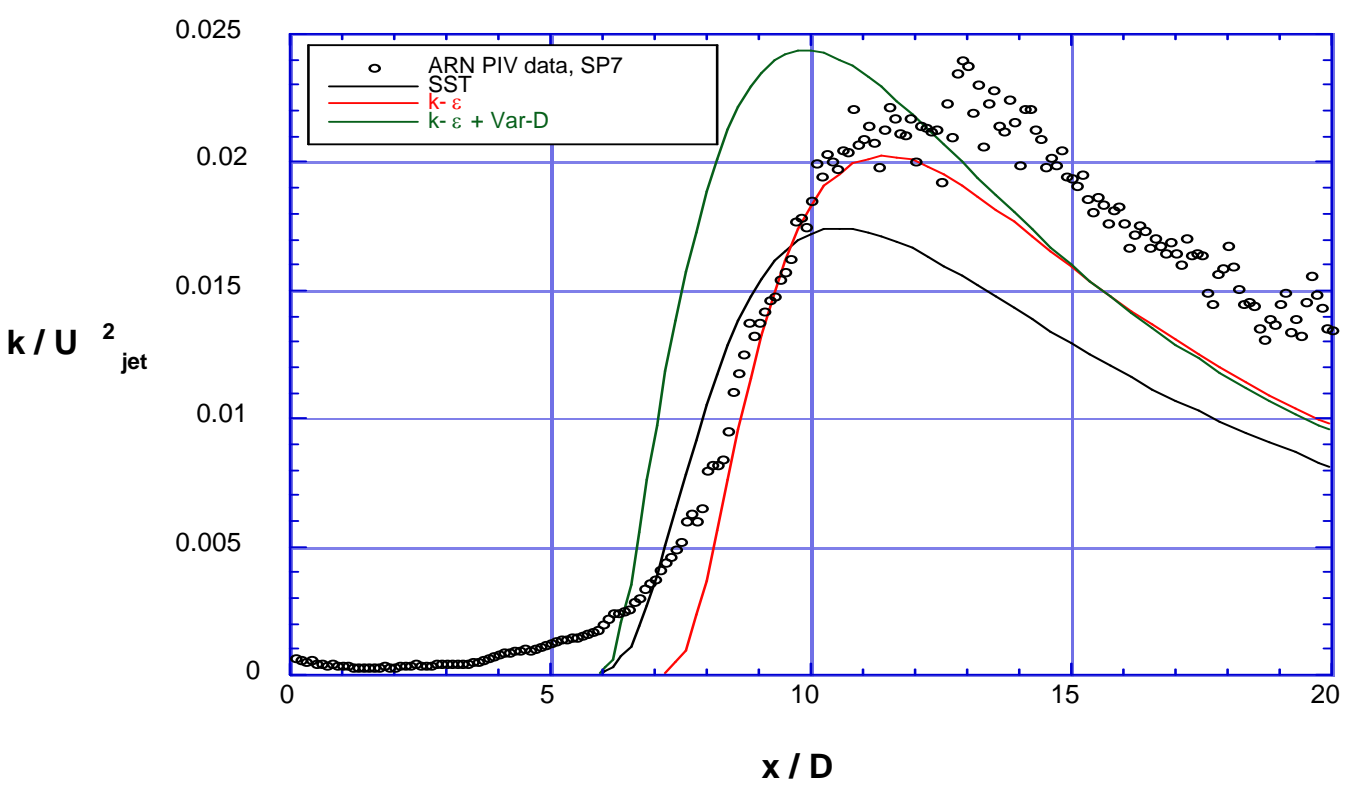

Fig. 14. Centerline turbulent kinetic energy for SP7 (near-sonic, unheated jet). 


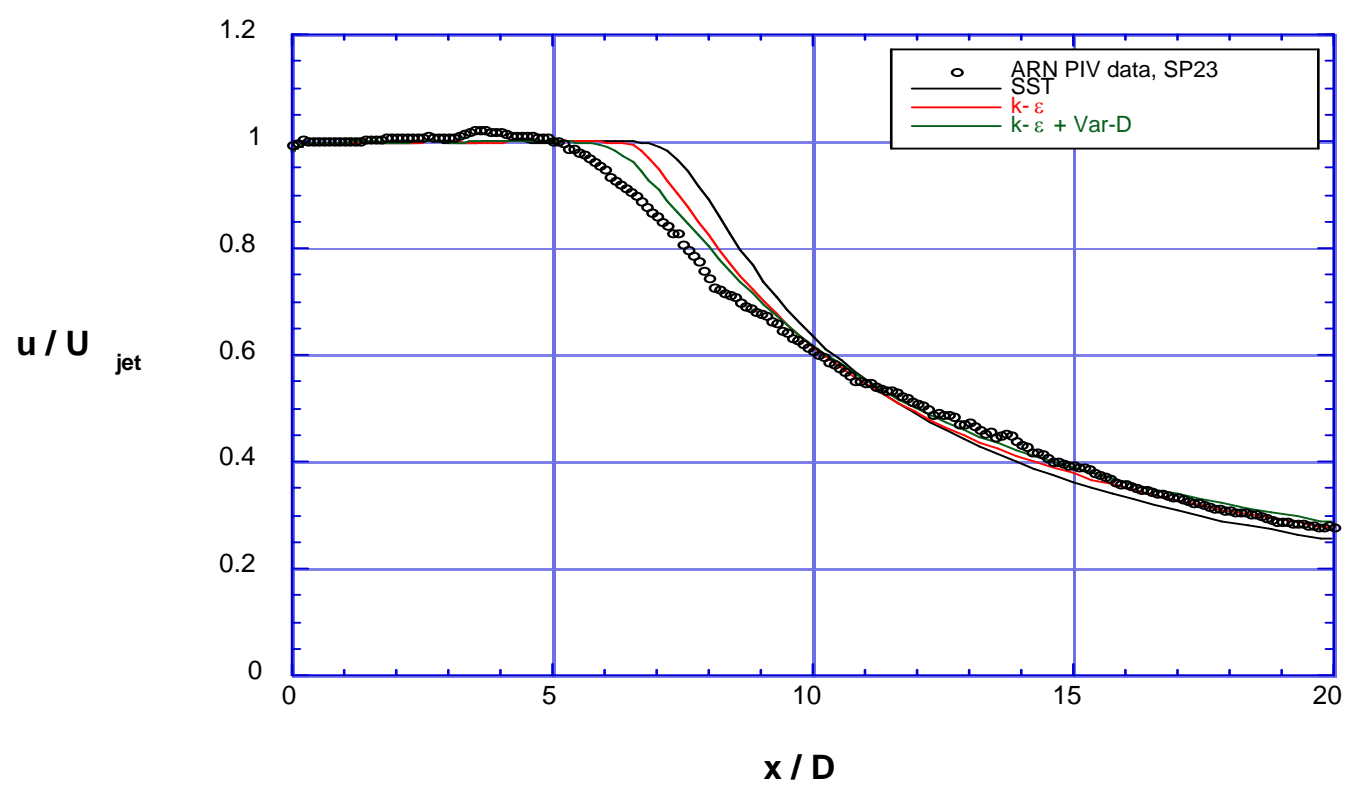

Fig. 15. Centerline axial velocity for SP23 (minimal compressibility, heated jet).

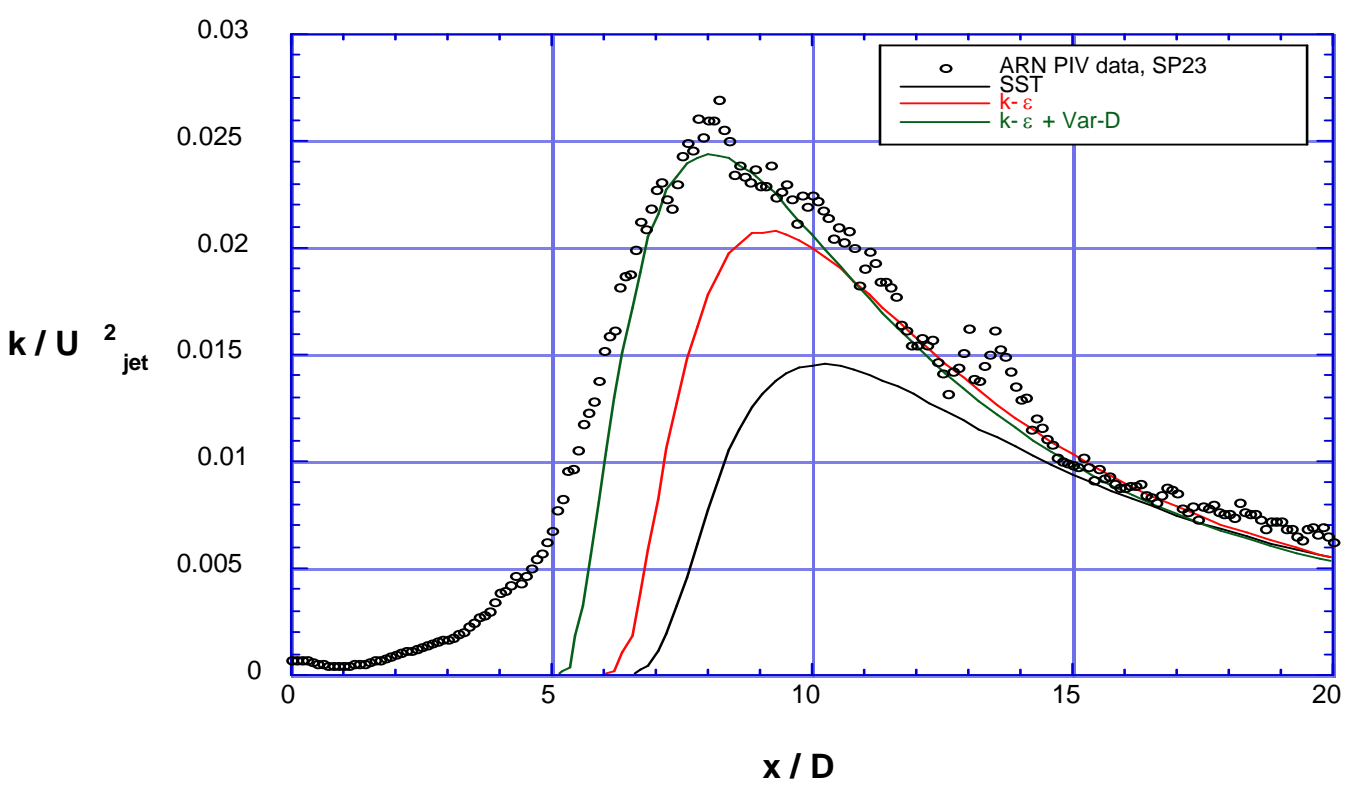

Fig. 16. Centerline turbulent kinetic energy for SP23 (minimal compressibility, heated jet). 


$$
\mathrm{K} / \mathrm{u}_{\mathrm{j}}^{2} \text { Levels } \begin{gathered}
0.030 \\
0.015 \\
0.000
\end{gathered}
$$

(a) ARN PIV data

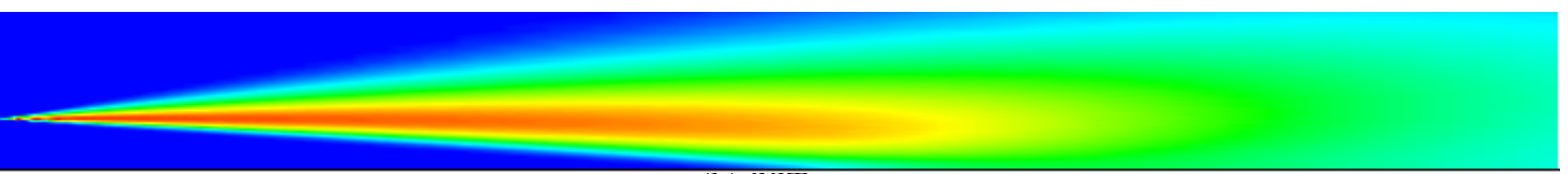

(b) SST

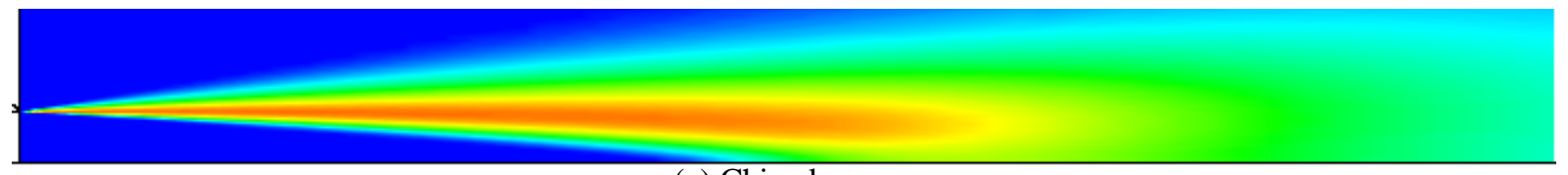

(c) Chien k- $\varepsilon$

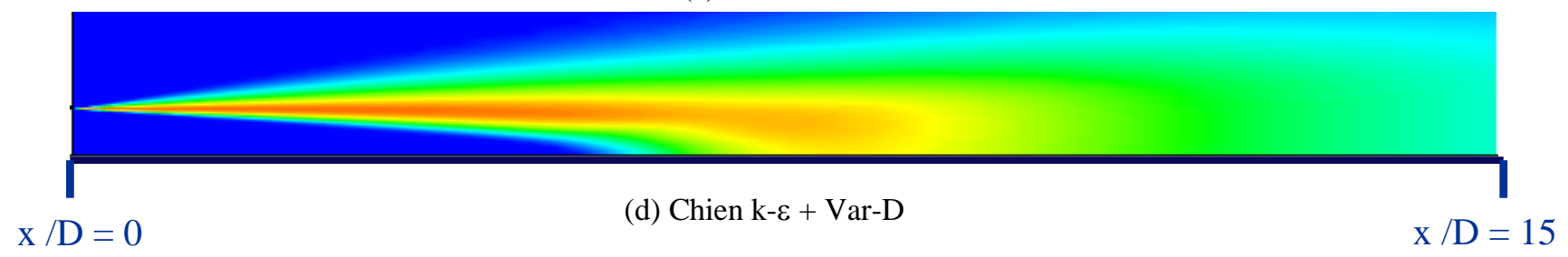

Fig. 17. Turbulent kinetic energy profiles for SP23 (minimal compressibility, heated jet). 


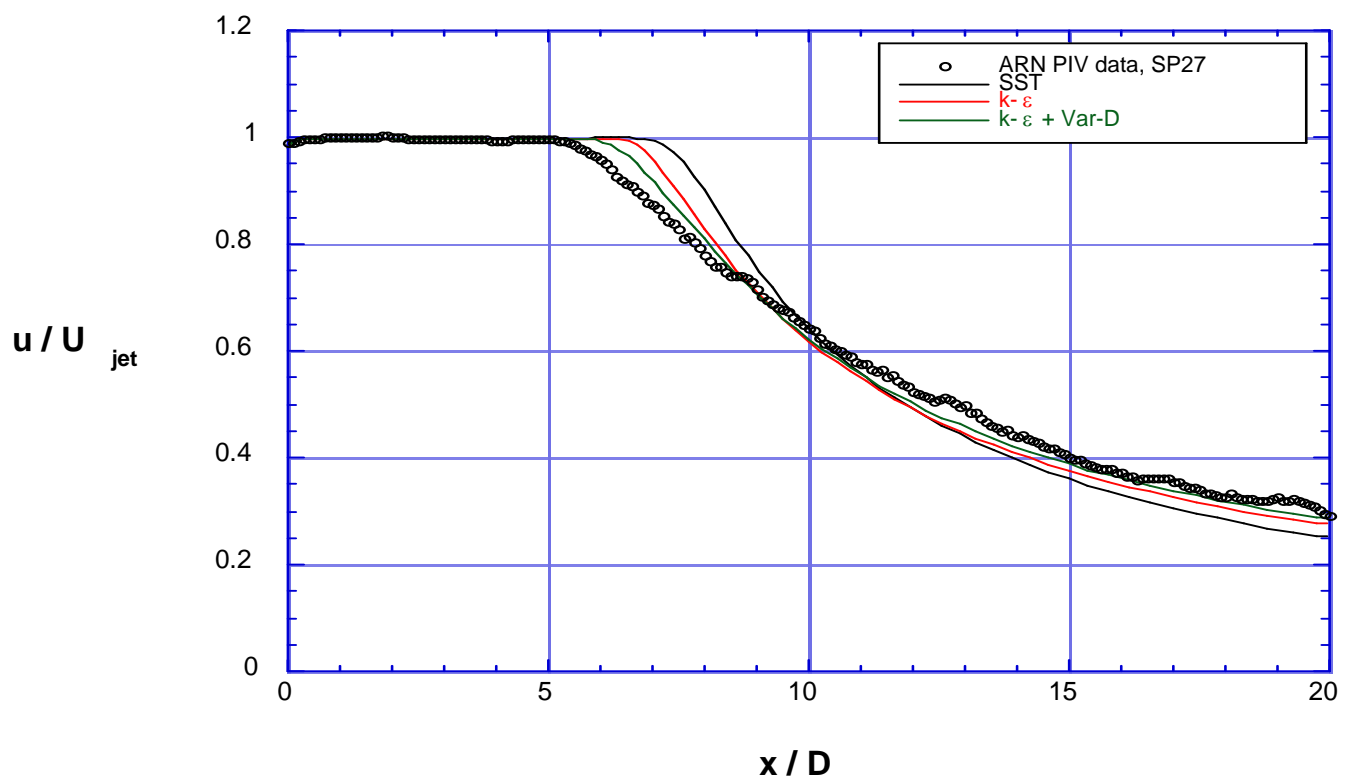

Fig. 18. Centerline axial velocity for SP27 (high-subsonic, heated jet).

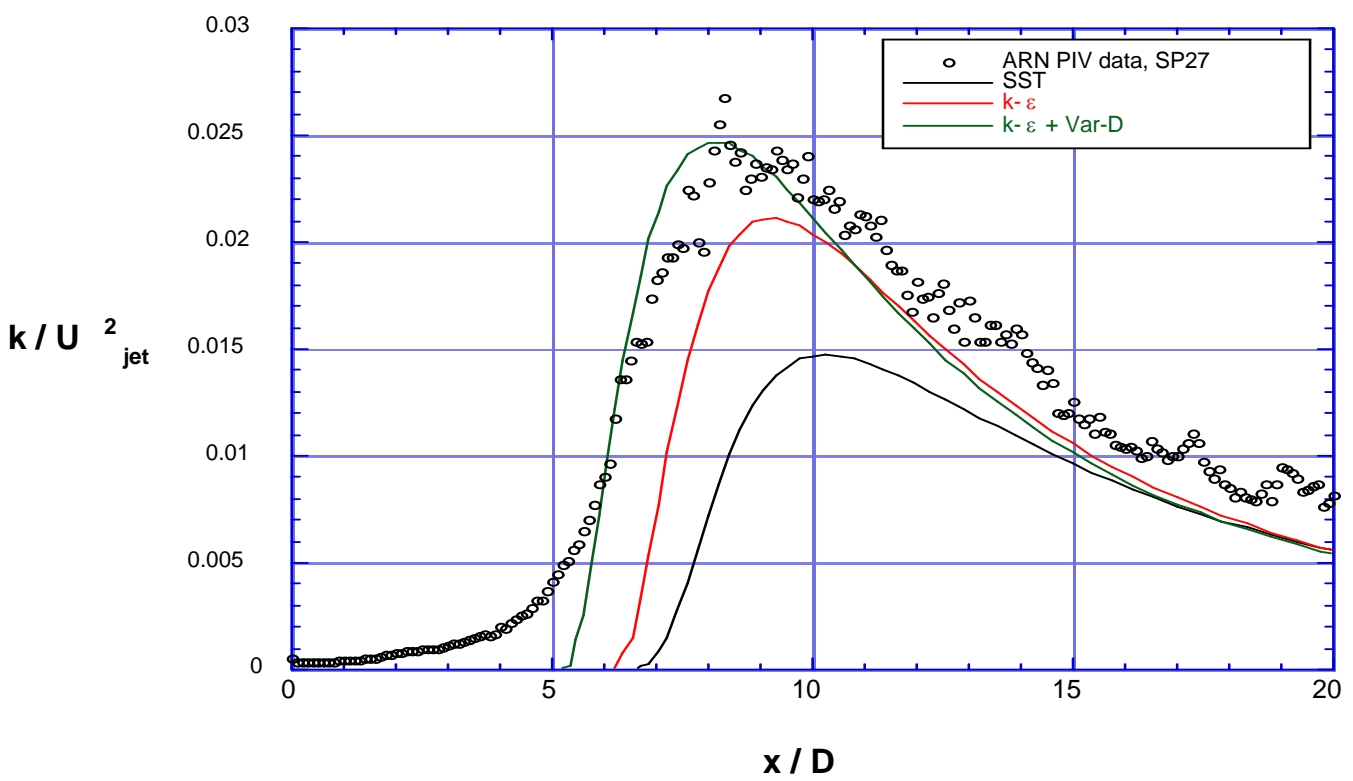

Fig. 19. Centerline turbulent kinetic energy for SP27 (high-subsonic, heated jet). 


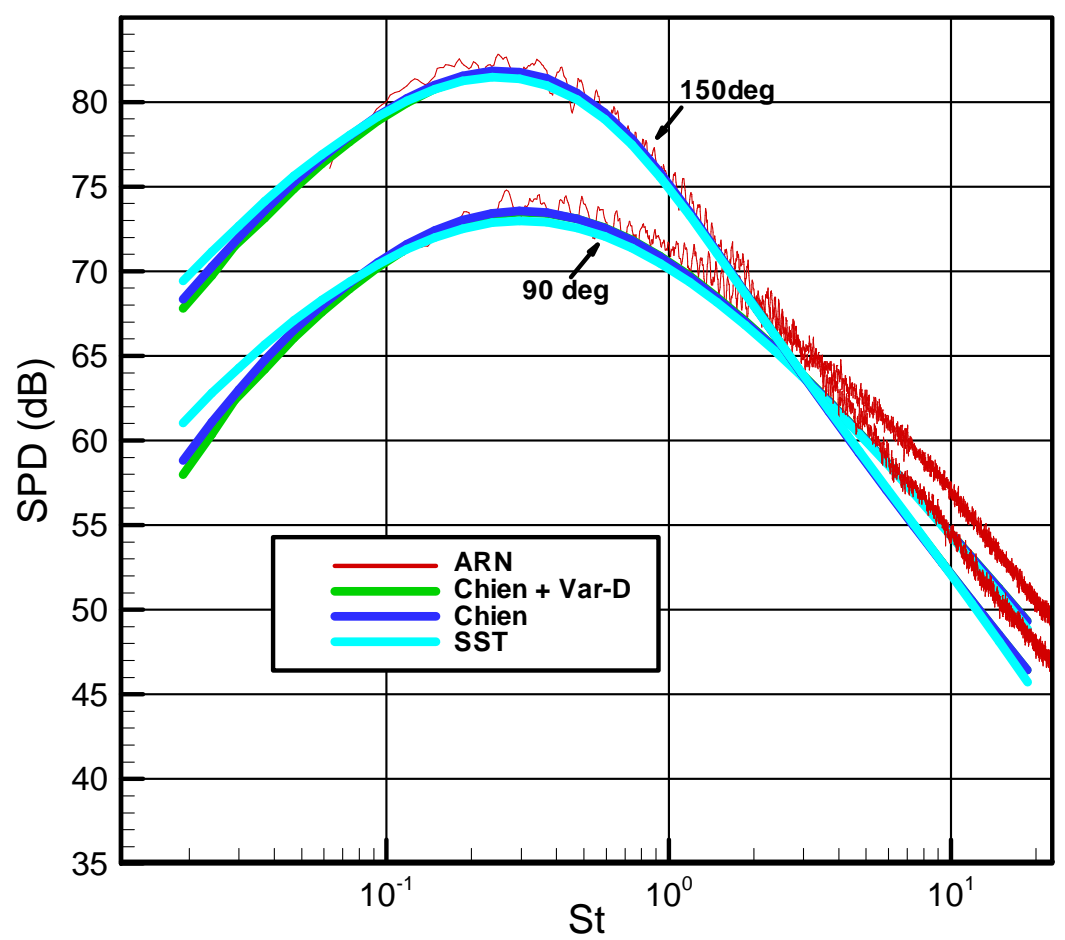

Fig. 20. Sound spectral density versus turbulence model variations for SP3 (low-subsonic, unheated jet).

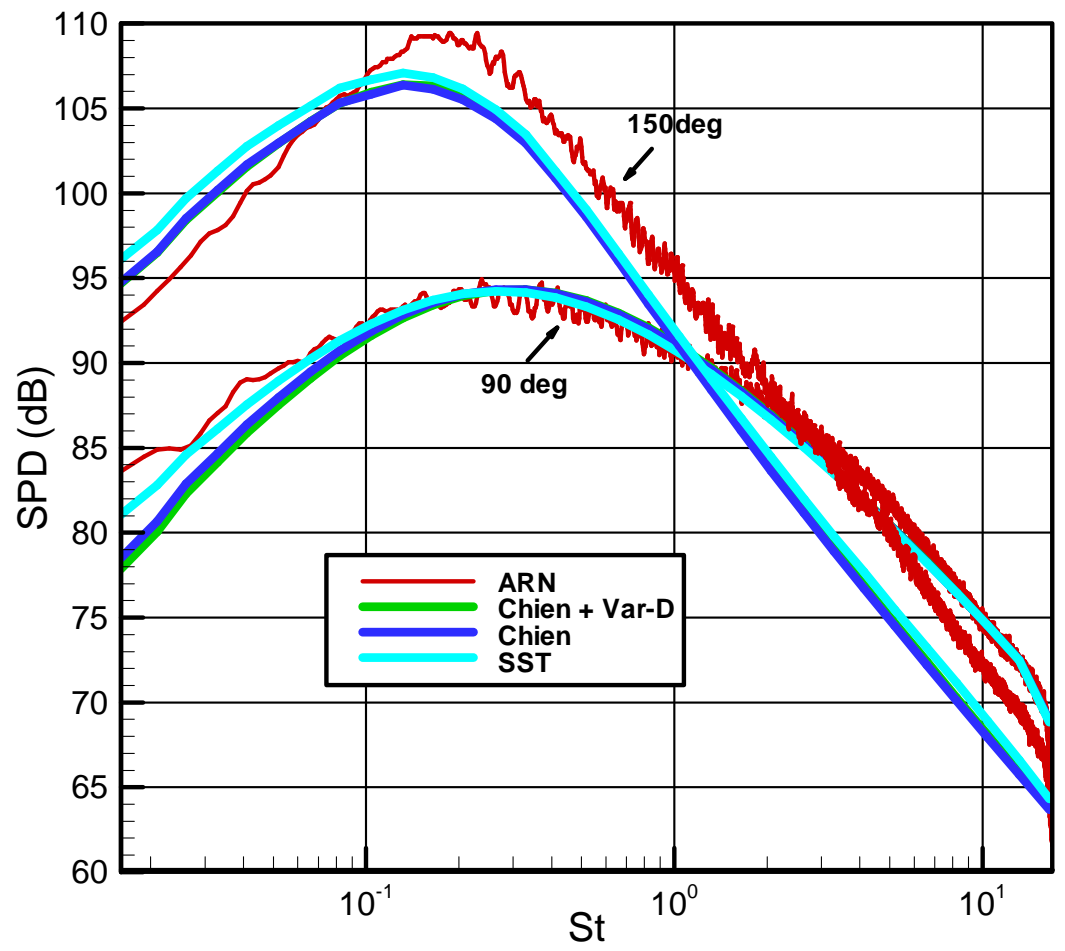

Fig. 21. Sound spectral density versus turbulence model variations for SP7 (high-subsonic, unheated jet). 